\title{
Effects of Low-Intensity Pulsed Ultrasound on the Proliferation of Human Umbilical Cord Mesenchymal Stem Cells and Their Chondrogenic Differentiation via Bmp2 /Smad Signaling Pathway
}

Xuhong JIN ( $\sim$ jxh53@sina.com )

Central South University

Hejie Wang

Central South University

Jiaju Che

Central South University

Xiafu Chen

Central South University

Dengao Huang

Central South University

Zeming Zhuo

Central South University

Shi Xing

Central South University

\section{Research Article}

Keywords: Low-intensity pulsed ultrasound, Umbilical cord mesenchymal stem cells, Bone morphogenetic protein, cell proliferation, chondrogensis

Posted Date: December 31st, 2020

DOI: https://doi.org/10.21203/rs.3.rs-122372/v1

License: (c) (i) This work is licensed under a Creative Commons Attribution 4.0 International License.

Read Full License 
Effects of low-intensity pulsed ultrasound on the proliferation of human umbilical

cord mesenchymal stem cells and their chondrogenic differentiation via BMP2 /smad signaling pathway

Hejie Wang†, Jiaju Che†, Xiafu Chen, Dengao Huang, Zeming Zhuo, Shi Xing, Xuhong Jin*.

*Correspondence: jxh53@sina.com

$\dagger$ Equal contributors

Orthopaedic Center, Affilated Haikou Hospital of Xianya Medical College, Central South University, Haikou 570208, China 


\section{Abstract}

Background: Although Low-intensity pulsed ultrasound (LIPUS) regiment has been applied to the treatment of cartilage injury clinically, the effect of LIPUS stress loading on the proliferation and differentiation of hUCMSCs is still controversial. Yet the specific mechanical mechanism of LIPUS stimulates cartilage differentiation of hUCMSCs has not been clarified.

Objective: To investigate the effects of low intensity pulsed ultrasound (LIPUS) on proliferation and chondrogenic differentiation of human umbilical cord mesenchymal stem cells (hUCMSCs) via BMP2 /smad signaling pathway in vitro.

Method: The hUCMSCs at P4 were randomly divided into control group(no LIPUS), $\mathrm{L}_{30}$ group $\left(30 \mathrm{~mW} / \mathrm{cm}^{2}\right), \mathrm{L}_{50}$ group $\left(50 \mathrm{~mW} / \mathrm{cm}^{2}\right), \mathrm{L}_{80}$ group $\left(80 \mathrm{~mW} / \mathrm{cm}^{2}\right)$, each LIPUS group respectively loaded $5,10,20 \mathrm{~min} / \mathrm{d}$, the cell proliferation activity was detected by CCK-8 assay after continuous irradiation for 5 days. For chondrogenic differentiation, hUCMSCs were divided 4 groups as follows: control group, Noggin group, LIPUS group, Noggin+LIPUS group, LIPUS irradiation was given $50 \mathrm{w} / \mathrm{cm}^{2}$, $5 \mathrm{~min} / \mathrm{d}$. Cartilage formation after 3 weeks was evaluated by Alician blue staining for GAG and COL II immunohistochemical staining. Furthermore, RT-PCR analysis of Sox-9, COL II Aggrecan, BMP2, Smad1, Smad5 and Smad9. Result : (1) The proliferation activity of L50 group $\left(50 \mathrm{w} / \mathrm{cm}^{2}, 5 \mathrm{~min} / \mathrm{d}\right)$ was noticeably higher compared 
with other groups, Alcian blue staining of GAG , COL II fluorescent staining in the LIPUS group were much stronger, quantitative RT-PCR assays showed that the expression of COL II, GAG, Sox-9, smad1, smad5 and smad9 in LIPUS group was significantly increased. Conclusion: The suitable LIPUS irradiation can promote cell proliferation, $50 \mathrm{~mW} / \mathrm{cm}^{2}$ intensity at $5 \mathrm{~min} / \mathrm{d}$ is most significant. And it can promote chondrogenesis of hUCMSCs via upregulating MP2 /smads signaling pathway.

Key word: Low-intensity pulsed ultrasound; Umbilical cord mesenchymal stem cells; Bone morphogenetic protein; cell proliferation; chondrogensis

\section{Introduction}

Stem cell based tissue engineering has emerged as a promising strategy for articular cartilage regeneration[1-4]. Human umbilical cord mesenchymal stem cells (hUCMSCs) derived from Wharton jelly tissues has the characteristics of stem cells and pre-chondrocytes, and naturally expresses Sox-9 and COL II positively, and it has the ability to differentiate and express multi-directional cells stably under specific conditions and stably express specific cell phenotypes. These characteristics make hUCMSCs an ideal seed cell for cartilage tissue engineering[5-8].

It is well known that stress loading is one of the most basic biological stimulation to cells in the process of biological evolution. MSCs can recruit, proliferate and 
differentiate into chondroblast under stress stimulation, which is the cytological basis of new cartilage formation[9-13]. In recent years, it has been found that Low intensity pulsed ultrasound intensity ultrasound (LIPUS) integrates mechanical stimulation of compressive stress and fluid shear stress through high frequency and small amplitude pulsed pressure wave. LIPUS direct act on joints can improve the nutrition of articular cartilage, reduce the production of inflammatory mediators, promote the repair of articular cartilage defects and promote the proliferation, directional differentiation and homing of stem cells in vitro and in Vivo. It has been gradually applied to the repair and healing treatment of cartilage injury clinically[14-15].

However, the effect of LIPUS stress loading on the proliferation and differentiation of hUCMSCs is controversial[16-20]. The suitable parameters of LIPUS including irradiation intensity and time need to be clarified. The specific mechanical mechanism of LIPUS stimulates cartilage differentiation and proliferation of hUCMSCs has not been fully elucidated.

Therefore, in our study, by evaluating the proliferation of hUCMSCs under different parameters of LIPUS loading conditions, suitable LIPUS loading conditions that could produce good chondrogenic differentiation effect on hUCMSCs in vitro were selected, and then the suitable parameter of LIPUS was loaded into hUCMSCs for chondrogenic differentiation and its effects through the BMP2 /Smad signaling 
pathway was further evaluated.

\section{Materials and methods}

2.1 The LIPUS exposure sonacell ultrasound device

The LIPUS Stimulation SonaCell Ultrasound Equipment (Intelligent-Nano,

Canada) is based on biological and clinical research. Its equipment parameters are:

Pulse Ultrasound Frequency 1.5 MHz, Pulse Repetition Frequency $1 \mathrm{KHz}$, Pulse Cycle $20 \%$. The average intensity of ultrasound can be adjusted to $0-100 \mathrm{~mW} / \mathrm{cm}^{2}$. The instrument probe is irradiated against the bottom of the culture plate through the coupling agent.

2.2 Cell culture and morphological observation

The P2 generation hUCMSCs(Saiye Biotechnology, Guangzhou,China) were adherent cultured in Dulbecco's modified Eagle's medium (DMEM; Saiye Biotechnology, Guangzhou,China) with 10\% fetal bovine serum (Saiye Biotechnology, Guangzhou,China), $100 \mathrm{mg} / \mathrm{mL}$ streptomycin, and $100 \mathrm{U} / \mathrm{mL}$ penicillin at $37^{\circ} \mathrm{C}$ in an incubator with $95 \%$ air and $5 \% \mathrm{CO}_{2}$. After $24 \mathrm{~h}$, the non-adherent cells were discarded by fluid exchange, and the fluid was changed every 2-3 days. When the cell fusion reached $80 \%$, the cells were digested by trypsin and passaged at the ratio of $1: 2$.

The cells were characterized morphologically under an inverted microscope (Olympus, Tokyo, Japan). Phenotype of hUCMSCs including CD29, CD34, CD44, 
CD45, CD73and CD105 was identified by flow cytometry, CD34 and CD45 are markers of haematopoietic stem cell.

\subsection{CCK-8 assay of cell viability}

P4 of hUCMSCs growing on 96-well plates at a density of $100 \mathrm{uL}$ with $1.5 \times 10^{4} / \mathrm{ml}$ were randomly divided into four groups: control group, L30 group $\left(30 \mathrm{~mW} / \mathrm{cm}^{2}\right), \mathrm{L} 50$ group ( $\left.50 \mathrm{~mW} / \mathrm{cm}^{2}\right), \mathrm{L} 80$ group $\left(80 \mathrm{~mW} / \mathrm{cm}^{2}\right)$. The LIPUS irradiation groups exposed the cell culture plate to the probe element of the SonaCell device through the coupling agent and placed it in the cell incubator for LIPUS stimulation at $5 \mathrm{~min} / \mathrm{d}, 10 \mathrm{~min} / \mathrm{d}$ or $20 \mathrm{~min} / \mathrm{d}$ respectively for 5 consecutive days, the control group was treated without LIPUS. And the culture medium was replaced after $24 \mathrm{~h}$.Another blank hole was taken and added into the culture medium as blank group. $10 \mu 1$ of CCK- 8 solution was added 24h, 48h, 72h, 96h and 120h later. Cells were cultured for an additional 4 hours and the absorbance at $450 \mathrm{~nm}$ was measured using a microplate reader. The proliferation inhibition rate $=(\mathrm{OD}$ of experimental group - OD of blank group $) /(\mathrm{OD}$ of control group - OD of blank group)x $100 \%$. Four groups of repeated experiments were performed. The data were statistically analyzed.

\subsection{Effect of LIPUS on Chondrogenic differentiation of hUCMSCs}

\subsubsection{Experimental grouping}

Adjust the cell density of P4 generation hUCMSCs to $1 \times 10^{5} / \mathrm{ml}$, inoculate in a 
6-well culture plate, and perform LIPUS stress loading $(50 \mathrm{~mW} / \mathrm{cm} 2,5 \mathrm{~min} / \mathrm{d}$.). The

hUCMSCs cultured with chondrogenic medium were divided into 4 groups as follow:

(A)control group: hUCMSCs were cultured in chondrogenic medium for 3 weeks;

(B)LIPUS group: hUCMSCs were given for LIPUS $\left(50 \mathrm{~mW} / \mathrm{cm}^{2}, 5 \mathrm{~min} / \mathrm{d}\right)$ stress

loading for 3 weeks; (C)Noggin group: hUCMSCs were added with BMPs antagonist

Noggin 50ng/ml treatment and then cultured for 3 weeks; (D)LIPUS+ Noggin group:

hUCMSCs were added BMPs antagonist Noggin 50ng/ml and LIPUS $\left(50 \mathrm{w} / \mathrm{cm}^{2}\right.$,

$5 \mathrm{~min} / \mathrm{d}$ )were continuously loaded for 3 weeks.

\subsubsection{Alcian blue staining}

After the hUCMSCs were cultured in chondrogenic medium for 3 weeks, the cells of each group were washed with PBS three times, fixed with 4\% paraformaldehyde at room temperature for $30 \mathrm{~min}$, PBS washed 3 times, $0.1 \mathrm{M} \mathrm{HCl}$ solution was adde for 5 minutes, $\mathrm{pH}$ value drops to $1.0 ; 1 \%$ alein blue staining for 30 minutes; PBS washed thrice.Then the cells were observed under an inverted microscope, and took pictures with Image-ProPlus software.

\subsubsection{Immunofluorescence staining}

After the hUCMSCs were cultured in chondrogenic medium for 3 weeks, and the cells were washed thrice with PBS, fixed with $4 \%$ paraformaldehyde at room temperature for $15 \mathrm{~min}$, and then washed with PBS three tims, treated with $0.5 \%$ 
Triton $\times-100$ transmembrane for $30 \mathrm{~min}$. PBS washed thrice, $0.5 \%$ bovine serum albumin (BSA) wet box at room temperature for 30min, antihuman primary antibodies against collagen II (dilution1:100; Abcam UK) were added and incubated at $4{ }^{\circ} \mathrm{C}$ overnight. After washing with PBS thrice, the fluorochromeconjugated secondary antibody $(1: 500 ; \mathrm{Abcam} \mathrm{UK}))$ were added and incubated at $37^{\circ} \mathrm{C}$ in the dark for $1 \mathrm{~h}$. Followed by washing thrice with PBS, counterstained with $1 \mathrm{ml}$ diamidine phenylindole (DAPI; Molecular Probes, Waltham, MA, USA) at $37^{\circ} \mathrm{C}$ for 30min. The cells were observed and counted under an inverted phase contrast fluorescence microscope (Olympus, Tokyo, Japan). Applying IPP 5.0 software to analyze the average value of Col-2 absorbance of each group of cells (average absorbance $=$ absorbance value/field of view $)$.

\subsubsection{Quantitative real-time (qRT)-PCR}

Total RNA was extracted using qRT-PCR kit (Tiangen Biotech, Beijing, China) following the manufacturer's instructions. RNA was converted into complementary DNA using an AMV RTase kit ((Tiangen Biotech, Beijing, China). PCR reaction body and reaction conditions are strictly according to the kit instructions,respectively detect Aggrecan, COL II, SOX-9, BMP2, smad1, smad5, smad9 gene expression. And according to the detected $\mathrm{Ct}$ value, calculate the mRNA expression level of each group of genes by $2-\Delta \Delta \mathrm{Ct}$ method $(\Delta \Delta \mathrm{Ct}=\Delta$ Ctsample $-\Delta$ Ctcalibrator). Primer pairs 
((Tiangen Biotech, Beijing, China) for target gene amplification are listed in Table 1.

Table I Fluorescence quantitative PCR primers.

\begin{tabular}{|c|c|c|}
\hline Genes & Forward primer $\left(5^{\prime} 3^{\prime}\right)$ & Reverse primer $\left(5^{\prime} 3^{\prime}\right)$ \\
\hline \multirow[t]{2}{*}{ Sox-9 } & A A A G A C C A C C C C GA T T A & T GAAGAT G G C G T T A G G \\
\hline & C AAG & A G A GA \\
\hline \multirow[t]{2}{*}{ Aggrecan } & A C C A C AAATGGAGCAG T & G G T C T G G T T G G G G T A G \\
\hline & T C A C C & A G G T A \\
\hline \multirow[t]{2}{*}{ COL II } & G C T C C C A GAACA T C A C C & T G G A C A G TA G AC G GA G \\
\hline & T A C C A C & GAAA G \\
\hline \multirow[t]{2}{*}{ BMP2 } & C G T GA G GAT T A G C A G G T & T C G C T T G A C G C T T T T C \\
\hline & C T T T G & T C T T C \\
\hline \multirow[t]{2}{*}{ Smad1 } & C C A G T T T G T T C T C C T T C & C G C C CT T C T TC T T C T T C \\
\hline & A T G A & A G T T \\
\hline \multirow[t]{2}{*}{ Smad5 } & G G G T T T GA G G C A GT G T A & G GA G C C CA T C T GA G T A \\
\hline & T G A G & A G G A C \\
\hline \multirow[t]{2}{*}{ Smad9 } & G C C C C T G C A C T G G T G C T & G C G T A C AC C T C G C C C C \\
\hline & $\mathrm{C} \mathrm{T} \mathrm{G}$ & $\mathrm{CAC}$ \\
\hline \multirow[t]{2}{*}{ GAPDH } & A GAGCACC A GA G GA G GA & T G G G A T G G A A A C T G T G \\
\hline & C G A & A A G A G G \\
\hline
\end{tabular}

\subsection{Statistical analysis}

All data were expressed as mean \pm standard deviation (SD) of three independent experiments and analyzed using SPSS 22.0 software (IBM Corp, Armonk, NY, USA). Multiple groups were compared by single-factor analysis of variance (ANOVA) and two groups by pair-wise Student's t test. $\mathrm{P}$ values $<0.05$ were considered statistically significant. 


\section{RESULTS}

3.1 General observation and characterization of hUCMSCs after LIPUS irradiation

The hUCMSCs were incubated under LIPUS stimulus for 5 days, the cells shaped

as fusiform and grew in vortex, more cell colonies are observed in the L50 group $\left(50 \mathrm{w} / \mathrm{cm}^{2}, 5 \mathrm{~min} / \mathrm{d}\right)$ than in the control group. As increased with the pulse intensity and the irradiation time, the cell colonies was decreased. Flow cytometry assay showed that the hUCMSCs were positive for CD29 (86.9 $\pm 3.73 \%)$, CD44 (99.91 $\pm 7.33 \%)$, CD73 $(95.84 .91 \pm 7.33 \%)$ and CD105 (87.99 $\pm 7.33 \%)$, and negative for CD34 (4.54 $\pm 0.83 \%)$ and CD45 $(1.19 \pm 0.88 \%)$.

\subsection{The effect of LIPUS irradiation on cell proliferation}

The results of hUCMSCs proliferation of each group after irradiating with different LIPUS intensities and duration time are shown(Fig. 1). At 5min/d, the cell proliferation rate of the $\mathrm{L} 50$ group was the highest $(\mathrm{P}<0.05)$. But the cell proliferation ability decreased with the increase of irradiation intensity and the pulse duration instead, cell proliferation is weakest at $\mathrm{L} 80$ group $\left(80 \mathrm{w} / \mathrm{cm}^{2}, 20 \mathrm{~min} / \mathrm{d}\right)(\mathrm{P}<0.05)$.
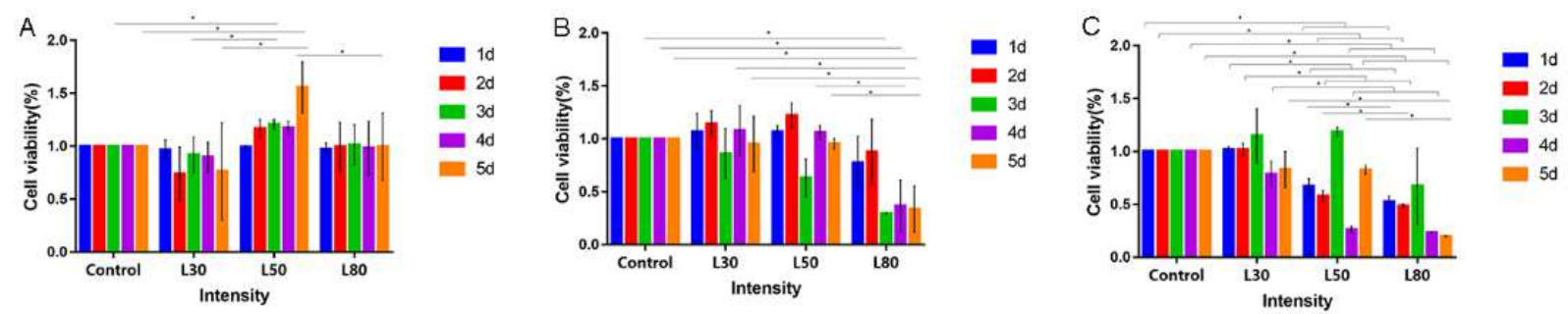
Fig.1 After culture for 24, 48, 72, 96, and 120 hours, the effect of LIPUS on hUCMSCs proliferation capacity was tested by CCK-8 assay at (A) $5 \mathrm{~min} / \mathrm{d}$, (B) $10 \mathrm{~min} / \mathrm{d}$, (C) $20 \mathrm{~min} / \mathrm{d}$. Note: $* \mathrm{P}<0 \cdot 05$.

\subsection{Effects of LIPUS irradiation on chondrogenic differentiation of hUCMSCs}

3.3.1 Immunohistochemical staining results of cartilage-specific proteins GAG, COL II and DAPI

After 3 weeks of chondrogenic induction, Alcian blue staining of GAG in the LIPUS group was much stronger than that in the control, Noggin, LIPUS+ Noggin groups (see Fig.2:A3-D3). The staining in Noggin group was weaker than that in the control group(Fig.2:C3). Immunofluorescence staining for COL II and DAPI results showed that COL II green-stained fluorescence distribution and nucleus blue were seen around and between the nuclei of each group(Fig.2:A1-D1). The COL II green staining fluorescent and the DAPI fluorescent staining of the blue stained fluorescent particles in LIPUS group $(\mathrm{P}=0.0054<0.01), \quad$ LIPUS + Noggin $\operatorname{group}(\mathrm{P}=0.0078<0.01)$ increased significantly compared with control group(Fig.2:A2-D2). But the expression of COL II in LIPUS + Noggin group $(\mathrm{P}=0.0419<0.01)$ was decreased than that of LIPUS group(Fig.2:C1,D1). 


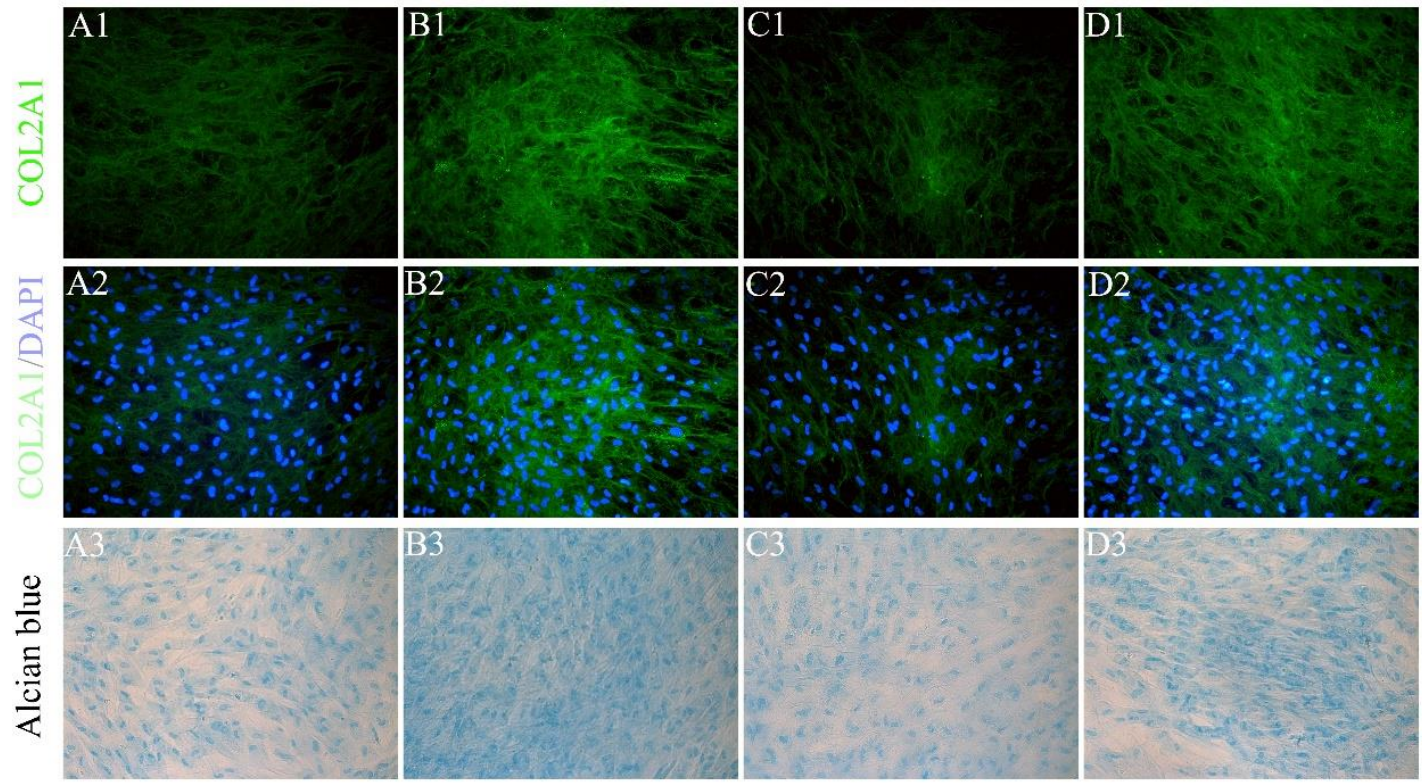

Fig.2 Immunofluorescence staining for COL II, DAPI and Alcian blue staining results are shown after 3 weeks of chondrogenic induction: Control group (A); LIPUS group (B); Noggin group (C); Noggin+LIPUS group (D).

\subsubsection{Quantitative RT-PCR detected the mRNA expression of COL II, GAG and Sox9}

After continuous in vitro chondrogenesis induction culture and LIPUS loading with hUMSCs for $3 \mathrm{w}$, The mRNA expression of COL II, GAG and Sox9 were detected by RT-PCR. Compared with the control group, the expression of COL II $(\mathrm{P}=$ $0.0187<0.05), \operatorname{GAG}(\mathrm{P}=0.0093<0.01)$ and $\operatorname{Sox} 9(\mathrm{P}=0.0042<0.01)$ were significantly increased in the LIPUS group. When hUCMSCs were stimulated with LIPUS $50 \mathrm{~mW} / \mathrm{cm}^{2}$ intensity and BMP2 specific blocker Noggin, the expression of COL II(P= $0.0212<0.05), \operatorname{GAG}(\mathrm{P}=0.019<0.05)$ and $\operatorname{Sox}-9(\mathrm{P}=0.0308<0.001)$ in LIPUS + Noggin group was significantly reduced than those of LIPUS group. However, after the addition of Noggin blocker, the expression of COL II $(\mathrm{P}=0.053>0.05), \mathrm{GAG}(\mathrm{P}=0.012<0.05)$ and Sox-9(P=0.033<0.05) in Noggin group was significantly decreased than control 
group.

3.3 Quantitative RT-PCR detected the mRNA expression levels of BMP2 signaling pathway related genes

Quantitative RT-PCR test results showed that the mRNA expression of $\mathrm{BMP} 2(\mathrm{P}=0.0172<0.05), \quad \operatorname{smad} 1(\mathrm{P}=0.0061<0.01), \quad \operatorname{smad} 5(\mathrm{P}=0.0166<0.05), \quad$ and $\operatorname{smad} 9(\mathrm{P}=0.0317<0.05)$ in the LIPUS group was significantly higher than those in control group, And the expression levels of $\operatorname{smad} 1(\mathrm{P}=0.005<0.01)$, $\operatorname{smad} 5(\mathrm{P}=0.018<0.05)$ and $\operatorname{smad} 9(\mathrm{P}=0.034<0.05)$ in the LIPUS +Noggin group was significantly less than those of LIPUS group, those in the Noggin group were the lowest among the experience group. But compared with the control group, $\operatorname{Smad} 1(\mathrm{P}=0.013<0.05)$ and $\operatorname{smad} 9(\mathrm{P}=0.009<0.05)$ in Noggin group are statistically significant increased, while there was no significant difference in $\operatorname{smad5}(\mathrm{P}=0.067>0.05)$ expression.

\section{DISCUSSION AND CONCLUSIONS}

At present, there is controversy about LIPUS irradiation can promote cell proliferation. Yoon $\mathrm{JH}$ et al has demonstrated that LIPUS can promote hUCMSCs proliferation, but no specific experimental screening of the radiation intensity parameters that promote cell proliferation was carried out [21]. On the contrary, Tien YC et al found that LIPUS stimulation did not promote the proliferation of 
chondrocytes [22]. Aliabouzar M et al study has shown that the optimum frequency of sound wave, pulse repetition and duty cycle are $1.5 \mathrm{Mhz}, 1 \mathrm{Khz}$ and $20 \%$ respectively[23]. Our experimental studies found that optical irradiation with $\left(50 \mathrm{w} / \mathrm{cm}^{2}\right.$, $5 \mathrm{~min} / \mathrm{d}$ ) intensity can significant promote the proliferation of hUCMSCs cells, but with the increase of irradiation intensity and the extension of irradiation time, the cell proliferation ability decreases instead, cell proliferation is obviously lowest with $\left(80 \mathrm{w} / \mathrm{cm}^{2}, 20 \mathrm{~min} / \mathrm{d}\right)$ intensity.

These results suggest that LIPUS irradiation has a "double-edged sword" effect to promote and inhibit cell proliferation. What are the reasons for the disparity in results between studies? We consider whether this is related to different pulse equipment parameters, pulse intensity, pulse action time, cell type, cell culture method, and number of cells seeded in different studies. So, further in vitro and in vivo experimental studies are needed for verification.

The effects of mechanical stimulation of LIPUS in cells are complex and interrelated. Numerous studies have demonstrated that BMP2, an important subtype of bone morphogenetic proteins (BMPs) of TGF $\beta$ superfamily, plays an important role in inducing MSCs to differentiate into cartilage. BMP2 mainly transmits the signal from the cell membrane to the nucleus by activating the $\mathrm{Smad} / 1 / 5 / 9$ proteins, thereby promoting the differentiation of cartilage and maintaining the phenotype of 
chondrocytes, chondrocytes lack of $\mathrm{Smad} / 1, \mathrm{Smad} / 5$ can cause severe dysplasia of cartilage. Noggin is a specific extracellular antagonist of BMP2 signaling, which can specifically bind BMP2 to prevent BMP2 from binding to its cell surface receptors, thereby inhibiting the effects of BMP2 biological function .

Our quantitative RT-PCR detection results showed that the expression of BMP2 and its key downstream signaling molecules Smad1, Smad5 and Smad9 in the LIPUS group were significantly up-regulated, and the expression levels of extracellular cartilage matrix GAG, COL II, and Sox9 were highest compared to the other groups after chondrogenic induction for $3 \mathrm{~W}$. When the exogenous BMP2 antagonist Noggin is added, the expression levels of COL II, GAG, and Sox-9 in the LIPUS+Noggin group is significantly reduced compared with the LIPUS group, and BMP2 and Smad1, Smad5, Smad9 were significantly down-regulated, indicating that Noggin can inhibit the chondrogenic differentiation of hUMSCs under the effect of LIPUS to a certain extent. These results suggested that the BMP2/smads signaling pathway has an important positive regulatory role in the process of hUMSCS chondrogenic differentiation under LIPUS irradiation.

We observed that the expression of COL II, GAG and Sox9, and the expression of BMP2, Smad1, Smad5 and Smad9 in the Noggin group were significantly lower than that of the LIPUS group, LIPUS+Noggin group. However, compared with the control 
group, the expression of smad1 and smad9 increased rather than decreased, while the expression of smad5 was no significant difference. Is it possible that the addition of the Noggin, an exogenous BMP2 inhibitor, may not completely inhibit the activation of BMP2? More work needs to be done to further explored. In addition, we found that the expression of Sox-9, COL II, and GAG in LIPUS+Noggin group was higher than the control group. Whether this may be related to the activation of other signaling pathways in the process of LIPUS promoting cartilage differentiation. More studies have verified that the interaction between multiple signaling pathways in the process of chondrogenic differentiation $[24,25]$.

In conclusion, LIPUS irradiation with suitable parameters can promotes the proliferation and chondrogenesis of hUMSCs, and the BMP2 /smads signaling pathway plays an important positive regulatory role in the process. Our findings uncover the mechanism of the effects of LIPUS on hUMSCs and provide some promising treatment for articular cartilage defects in the future.

\section{Declarations:}

\section{Ethics approval and consent to participate:}

The experimental protocol was established, according to the ethical guidelines of the Helsinki Declaration and was approved by the Human Ethics Committee of 
Affilated Haikou Hospital of Xianya Medical College. Written informed consent was obtained from individual or guardian participants.

\section{Consent for publication:}

Not applicable.

\section{Competing interests}

The authors declare that they have no competing interests.

\section{Funding}

This study was supported in part by grants from Hainan Province Natural Science fundation (20158297).

\section{Authors' contributions}

HJW and XHJ participated in study design conducted all experiments and sample analysis. XFC and JJC conceived this study and participated in the design of the experiments, provided assistance for data analysis and statistical analysis and reviewed the manuscript. ZMZ and SX participated in the development of the study design and data analysis. DGH contributed to the technical work of the model used in the study.

All authors read and approved the final manuscript.

\section{Acknowledgments}

This study was financially supported by a grant from Hainan Province Natural Science fundation (20158297). 


\section{Author details}

Orthopaedic Center, Affilated Haikou Hospital of Xianya Medical College, Central

South University, Haikou 570208, China

\section{References}

[1] HanY, Li XZ, ZhangYB, Han YP, Chang F, Ding JX. Mesenchymal stem cells for regenerative medicine. Cells. 2019;8(8):886.

[2] Confalonieri D, Schwab A, Walles H, Ehlicke F. Advanced Therapy Medicinal Products: A guide for bone marrow-derived MSC application in bone and cartilage tissue engineering. Tissue Eng Part B Rev.2018;24(2):155-69.

[3] Lopez-Alcorocho JM, Aboli L,Guillen-Vicente I, Rodriguez-Iñigo E, GuillenVicente M, Fernández-Jaén TF, et al. Cartilage defect treatment using highdensity autologous chondrocyte implantation: two-year follow-up. Cartilage. 2018;9(4):363-9.

[4] Nixon AJ, Sparks HD, Begum L, McDonough S, Scimeca MS, Moran N, et al. Matrix-induced autologous chondrocyte implantation (MACI) using a cellseeded collagen membrane improves cartilage healing in the equine model. J Bone Joint Surg Am. 2017;99(23):1987-98. 
[5] Marmotti A, Mattia S, Castoldi F, Barbero A, Mangiavini L, Bonasia DE, et al. Allogeneic umbilical cord-derived mesenchymal stem cells as a potential source for cartilage and bone regeneration: an in vitro study. Stem Cells Int. 2017(1):116.

[6] Sadlik B, Jaroslawski G, Puszkarz M, Blasiak A, Oldak T, Gladysz D, et al. Cartilage repair in the knee using umbilical cord Wharton's jelly-derived mesenchymal stem cells embedded onto collagen scaffolding and implanted under dry arthroscopy. Arthrosc Tech. 2017;7(1):e57-e63.

[7] Zhang Y, Liu S, Guo W, Wang M, Hao C, Gao S, et al. Human umbilical cord Wharton's jelly mesenchymal stem cells combined with an acellular cartilage extracellular matrix scaffold improve cartilage repair compared with microfracture in a caprine model. Osteoarthritis Cartilage.2018;26(7):95465.

[8] Contentin R, Demoor M, Concari M, Desancé M, Audigié F, Branly T, et al. Comparison of the Chondrogenic Potential of Mesenchymal Stem Cells Derived From Bone Marrow and Umbilical Cord Blood Intended for Cartilage Tissue Engineering. Stem Cell Rev Rep. 2020;16(1):126-43. 
[9] Choi JR, Yong KW, Choi JY. Effects of mechanical loading on human mesenchymal stem cells for cartilage tissue engineering. J Cell Physiol.2018;233(3):1913-28.

[10] DeFrate LE, Kim-Wang SY, Englander ZA, McNulty AL. Osteoarthritis year in review 2018: mechanics. Osteoarthritis Cartilage. 2019;27(3):392-400.

[11] Steward AJ, Kelly DJ. Mechanical regulation of mesenchymal stem cell differentiation. J Anat. 2015,227(6):717-31.

[12] Kwon HJ, Yasuda K. Chondrogenesis on sulfonate-coated hydrogels is regulated by their mechanical properties. J Mech Behav Biomed Mater. 2013;17:337-46.

[13] Ando W, Fujie H, Moriguchi Y, Nansai R, Shimomura K, Hart DA, et al. Detection of abnormalities in the superficial zone of cartilage repaired using a tissue engineered construct derived from synovial stem cells. Eur Cell Mater. 2012;24: 292-307.

[14] Rothenberg JB, Jayaram P, Naqvi U, Gober J, Malanga GA. The Role of LowIntensity Pulsed Ultrasound on Cartilage Healing in Knee Osteoarthritis: A Review. PM R. 2017;9(12): 1268-77.

[15] Zhou XY, Zhang XX, Yu GY, Zhang ZC, Wang F, Yang YL, et al. Effects of Low-Intensity Pulsed Ultrasound on Knee Osteoarthritis: A Meta-Analysis of Randomized Clinical Trials. BioMed Research International, 2018(2):1-7. 
[16] Uddin SM, Richbourgh B, Ding Y, Hettinghouse A, Komatsu DE, Qin YX, et al.

Chondro-protective effects of low intensity pulsed ultrasound. Osteoarthritis Cartilage. 2016;24(11):1989-98.

[17] Kim ED, Won YH, Park SH, Seo JH, Kim DS, Ko MH, et al. Efficacy and Safety of a Stimulator Using Low-Intensity Pulsed Ultrasound Combined with Transcutaneous Electrical Nerve Stimulation in Patients with Painful Knee Osteoarthritis. Pain Res Manag. 2019(5):1-10.

[18]Yang SW, Kuo CL, Chang SJ, Chen PC, Lin YT, Manousakas L, et al. Does Low-intensity pulsed ultrasound treatment repair articular cartilage injury? A rabbit model study. 2014;15(1):36.

[19] Rothenberg JB, Jayaram P, Naqvi U, Gober J, Malanga GA. The Role of LowIntensity Pulsed Ultrasound on Cartilage Healing in Knee Osteoarthritis: A Review. PM R. 2017;9(12):1268-77.

[20] Wang X, Lin Q, Zhang T, Wang XW, Cheng K, Gao MX, et al. Low-intensity pulsed ultrasound promotes chondrogenesis of mesenchymal stem cells via regulation of autophagy. Stem Cell Res Ther. 2019;10(1):41.

[21] Yoon JH, Roh EY, Shin S,Jung NH, Song EY, Lee DS, et al. Introducing pulsed low-intensity ultrasound to culturing human umbilical cord- derived mesenchymal stem cells[J]. Biotechnol Lett. 2009;31(3): 329-35. 
[22] Tien YC, Lin SD, Chen CH, Lu CC, Su SJ, Chih TT. Effects of pulsed low-intensity ultrasound on human child chondrocytes. Ultrasound Med Biol. 2008;34(7): 1174-81.

[23] Aliabouzar M, Zhang LG, Sarkar K. Lipid Coated Microbubbles and Low Intensity Pulsed Ultrasound Enhance Chondrogenesis of Human Mesenchymal Stem Cells in 3D Printed Scaffolds. Sci Rep. 2016;6: 37728.

[24] Nishimura R, Hata K, Nakamura E, Murakami T, Takahata Y. Transcriptional Network Systems in Cartilage Development and Disease. Histochem Cell Biol. 2018;149(4):353-63.

[25] Chijimatsu R, Saito T. Mechanisms of synovial joint and articular cartilage development. Cell Mol Life Sci. 2019;76(20):3939-52. 

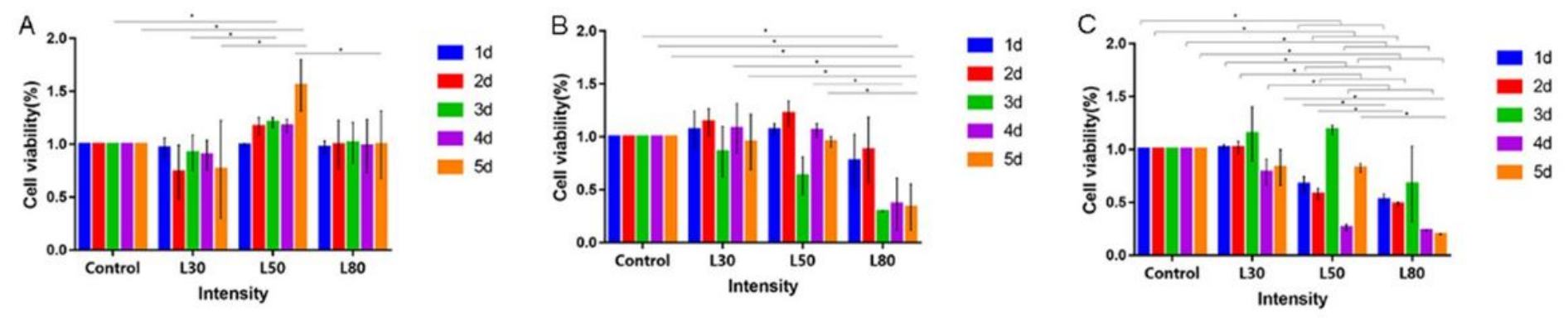

Figure 1

After culture for $24,48,72,96$, and 120 hours, the effect of LIPUS on hUCMSCs proliferation capacity was tested by CCK囚8 assay at $\triangle A \otimes 5 \mathrm{~min} / \mathrm{d}$, (B) $10 \mathrm{~min} / \mathrm{d}$, (C) $20 \mathrm{~min} / \mathrm{d}$. Note: $* \mathrm{P}<0.05$.
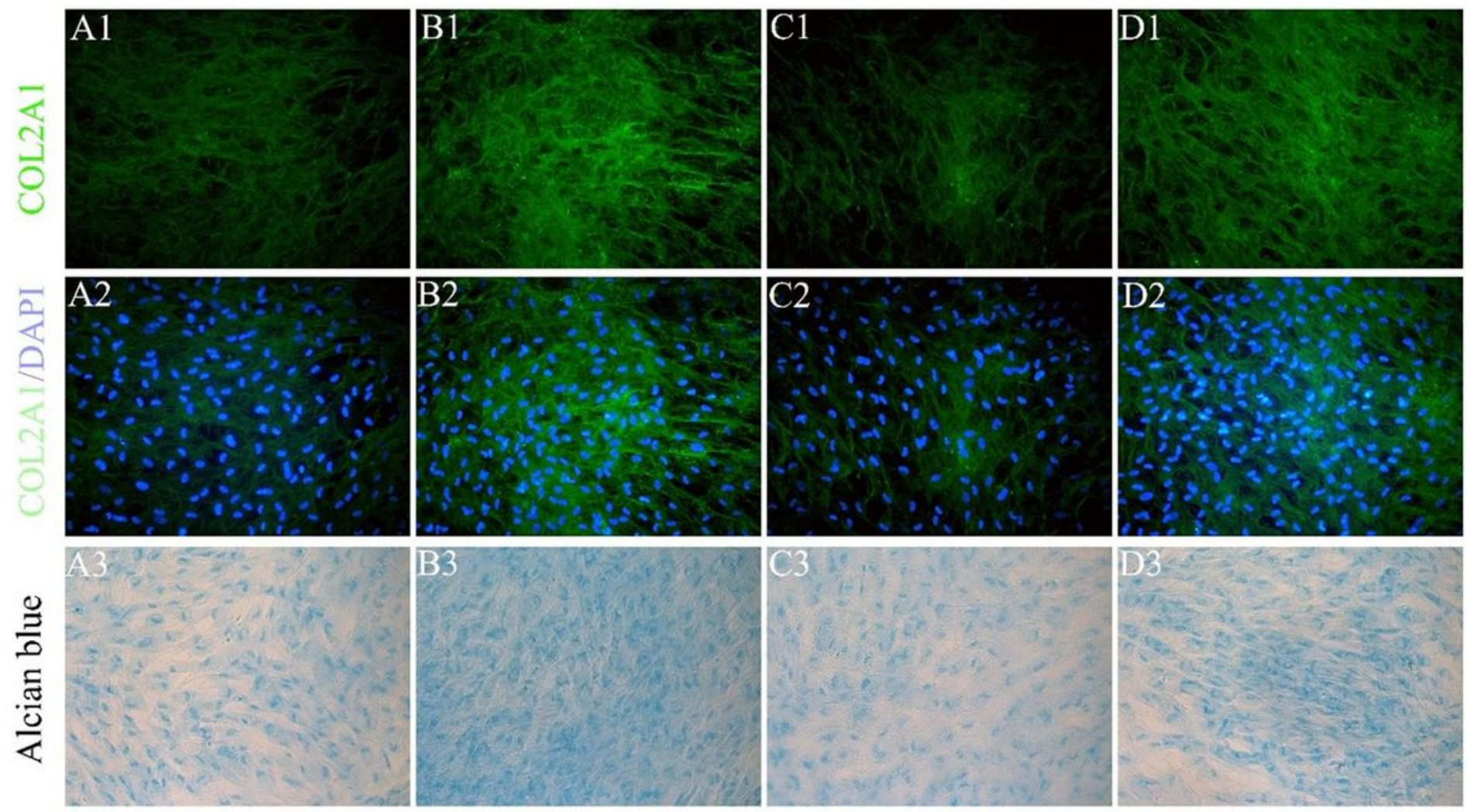

Figure 2

Immunofluorescence staining for COL II, DAPI and Alcian blue staining results are shown after 3 weeks of chondrogenic induction: Control group (A); LIPUS group (B); Noggin group (C); Noggin+LIPUS group (D). 